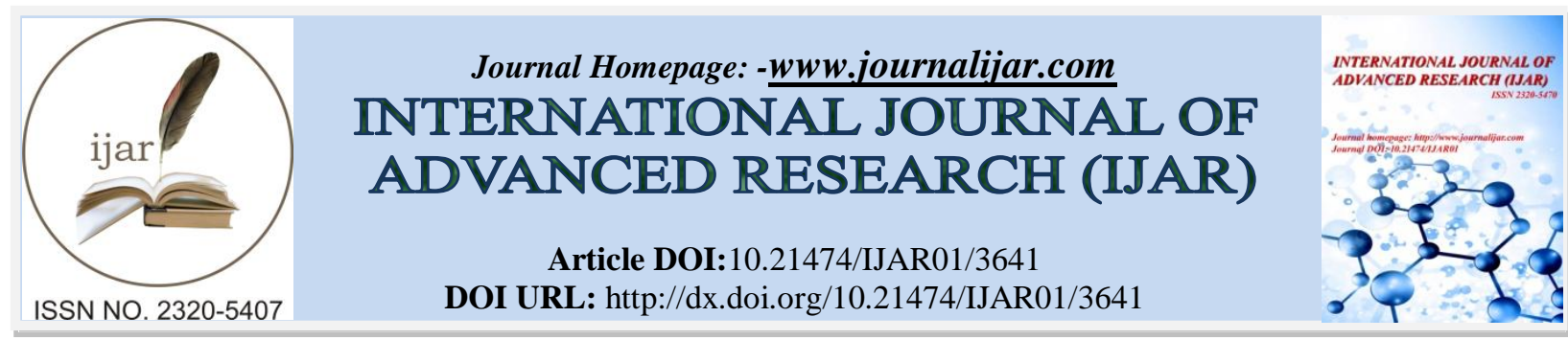

RESEARCH ARTICLE

\title{
ACUTE EOSINOPHILIC APPENDICITIS PRESENTING BY LOWER GASTRO-INTESTINAL BLEEDING.
}

\section{Basim Abdulrazzak Felemban ${ }^{1}$, Suleiman Jastaniah ${ }^{2}$, Nawaf H. Fatani ${ }^{3}$, Sawsan A. Khan ${ }^{3}$ and Amal Ali Hassan $^{4}$.}

1. Consultant Interventional Radiologist and Abdominal Imaging - Al-Noor Specialist Hospital, Makkah, K.S.A.

2. Associate Prof. of Surgery - Medical College Umm Al-Qura University, Consultant General Surgery - Al-Noor Specialist Hospital, Makkah, K.S.A.

3. MBBS, Faculty of Medicine, Umm Al-Qura University, Makkah, K.S.A.

4. Assistant Prof. Histopathology Faculty of Medicine, AlAzharUniversity, Consultant Histopathology Al-Noor Specialist Hospital, Makkah, K.S.A.

\section{Manuscript Info}

Manuscript History

Received: 08 January 2017

Final Accepted: 10 February 2017

Published: March 2017

Key words:-

Appendicitis, Eosinophil, Lower gastrointestinal bleeding.

\section{Abstract}

Acute eosinophilic appendicitis is a rare type of appendicitis where the inflamed appendix infiltrates by eosinophil rather than neutrophil in the mascularispropria accompanied by edema. Associated with type 1 hypersensitivity that may affect any part of the gastrointestinal track. Acute eosinophilic appendicitis similar to the classical appendicitis in symptoms. However, massive lower gastrointestinal bleeding with appendiceal origin considers a rare presentation of acute appendicitis. Different modalities are needed to reach the definitive diagnosis and identification of the bleeding site. We report a 51-year-old with appendiceal hemorrhage due to acute eosinophilic appendicitis. The Diagnosis was made by a multidisciplinary approach. The patient underwent upper and lower endoscopy, which wasinconclusive. Therefore a computerized tomography scanwas done to the patient to identify the source of bleeding. A right hemicolectomy was performed and the histopathology showed an acutely inflamed appendix with eosinophilic infiltration.

Copy Right, IJAR, 2017,. All rights reserved.

\section{Introduction:-}

Acute appendicitis is one of the most common causes of acute abdomen that requires surgical intervention [1]. The lifetime risk of developing acute appendicitis in Western populations is $7 \%$ [4]. It is a clinical-based condition where there is right iliac fossa pain and tenderness, vomiting, fever and high white blood cell (WBC) count [2,3]. The typical histopathologic finding of acute appendicitis is neutrophil infiltration of muscularispropria [2]. Eosinophil infiltration of the musclaris is considered a rare histopathologiccause of acute appendicitis [4]. Acute eosinophilic appendicitis(AEA) is still a not well-studied disease, and few cases were reported [5].

Lower Gastrointestinal Bleeding (LGIB) caused by acute appendicitis is extremely rare [6]. In this case report, the patient presented by severe LGIB as the main manifestation caused by acute eosinophilic appendicitis. The objective of this case report is to document two rare entities: Acute Eosinophilic Appendicitis and severe LGIB caused by appendicitis. 


\section{Case Presentation:-}

A 51-year-old Saudi male patient known case of hypothyroidism on Levothyroxine ( $250 \mathrm{mcg} / \mathrm{day})$, He presented to the emergency department of a general hospital due to several attacks of diarrhea, passing dark colored stool, for one day. The last attack of diarrhea ended by passing bright red blood per rectum and was associated with loss of consciousness. In the hospital, he was vitally stable and afebrile; the abdomen was soft and lax with positive bowel sounds. The patient denied any history of fever, nausea, vomiting or abdominal pain.

The initial lab work revealed a hemoglobin $(\mathrm{Hb})$ level of $12 \mathrm{~g} / \mathrm{dl}$, white blood cell (WBC) count of 15.3 and platelet count 265,000. The chemistry including liver function tests (LFTs) were within normal.

While in the emergency department, the patient had two episodes of per rectal bleeding. Repeated lab work revealed a severe drop of $\mathrm{Hb}$ level to $7.5 \mathrm{~g} / \mathrm{dl}$. The patient was shifted to ICU, received two units of Packed RBCs. Urgent upper GI endoscopy was performed without any abnormality detected. A lower GI endoscopy confirmed the presence of fresh blood in the colon; however, the source of the bleeding was not determined.

The patient was transferred to our center for further evaluation by CT angiography of the abdomen and pelvis (GI bleeding protocol) and for possible endovascular embolization.

The CT demonstrated no active bleeding from both upper and lower GI. However; the appendix was increased in diameter $(12 \mathrm{~mm}$ ) with mucosal hyper enhancement, diffuse mural thickening, peri-appendicular fat stranding (Fig. 1 and 2) and multiple ileocecal lymph nodes (Fig. 3). Near the tip of the cecum/the origin of the appendix, focal mural thickening was also noted (Fig. 4 and 5).

Another lower GI endoscopy was done in order to perform an endoscopic biopsy. The performing gastroenterologist was able to reach the ascending colon but was unable to reach the cecum. Again, blood clots were demonstrated in the ascending colon.

General surgery consultation was requested. Due to the focal thickening in the tip of the cecum, there was a serious concern for malignancy, which would explain theLGIB. Virtual CT colonoscopy was performed to further evaluate the cecum, as this was not fully evaluated by the conventional colonoscopy. The findings were more or less the same as the first CT study. A decision was made to perform a right hemicolectomy due to possible underlying malignancy. The histopathology of the specimen revealed an acutely inflamed appendix with transmural eosinophil cell infiltration. There were abundant luminal exudates and widespread mucosal ulceration. The included lymph nodes showed only reactive hyperplasia (Fig. 6).

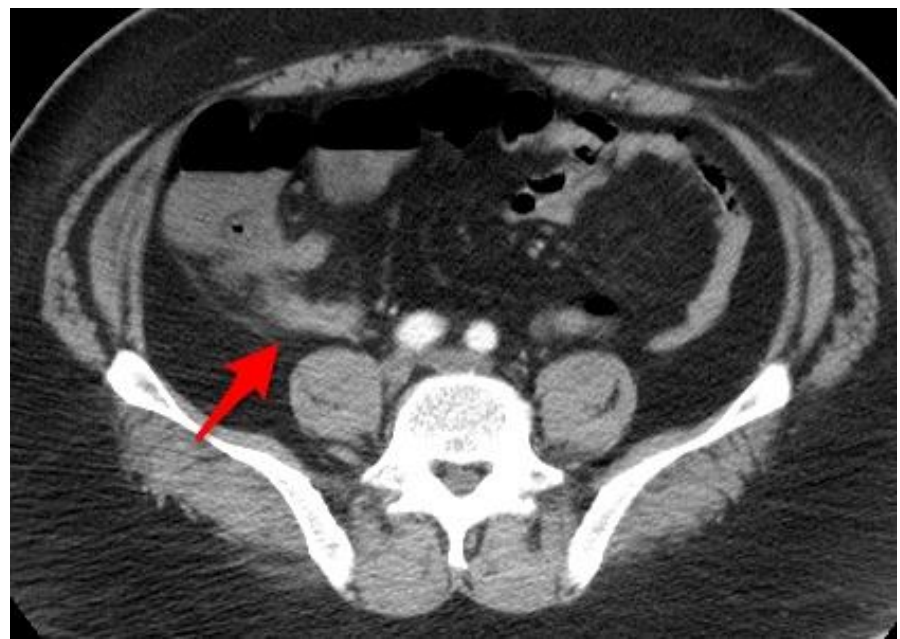

Fig (1):-CT abdomen and Pelvis, axial view, arterial phase: Circumferential mural thickening of the appendix with mucosal hyperenhancement (red arrow). There is also mild periappendicular fat stranding as shown. The maximum diameter of the appendix is $12 \mathrm{~mm}$ (measurement not shown). 


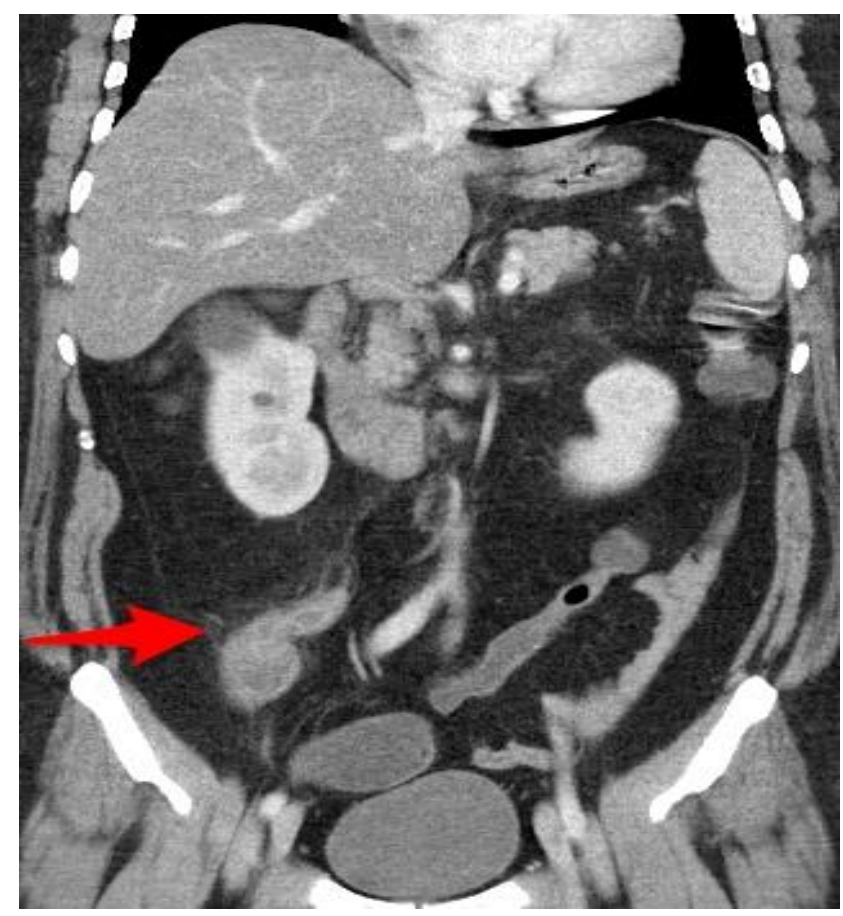

Fig (2):- CT abdomen and Pelvis, Coronal Reformats, portal venous phase: Circumferential mural thickening of the appendix with mucosal hyperenhancement (red arrow). There is also mild peri-appendicular fat stranding as shown. The maximum diameter of the appendix is $12 \mathrm{~mm}$ (measurement not shown).

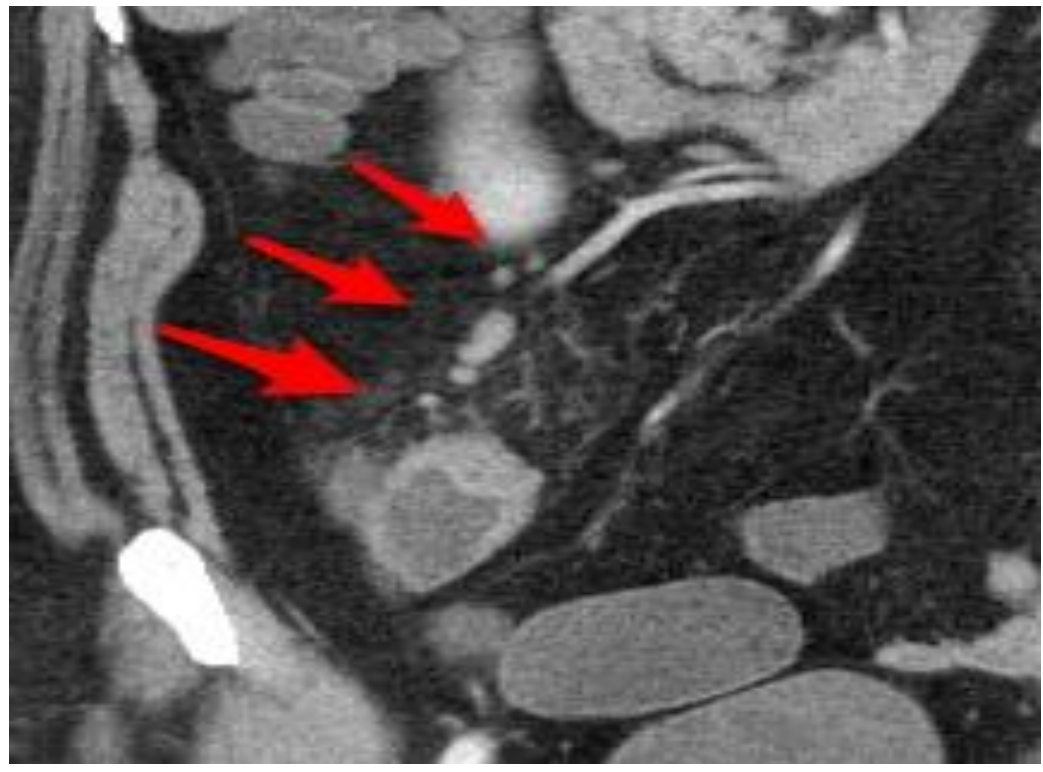

Fig (3):- CT abdomen and Pelvis, Coronal Reformats, portal venous phase, Focused on Ileocecal region: multiple small regional lymph nodes with meso-appendicular fat straning (multiple red arrows) 


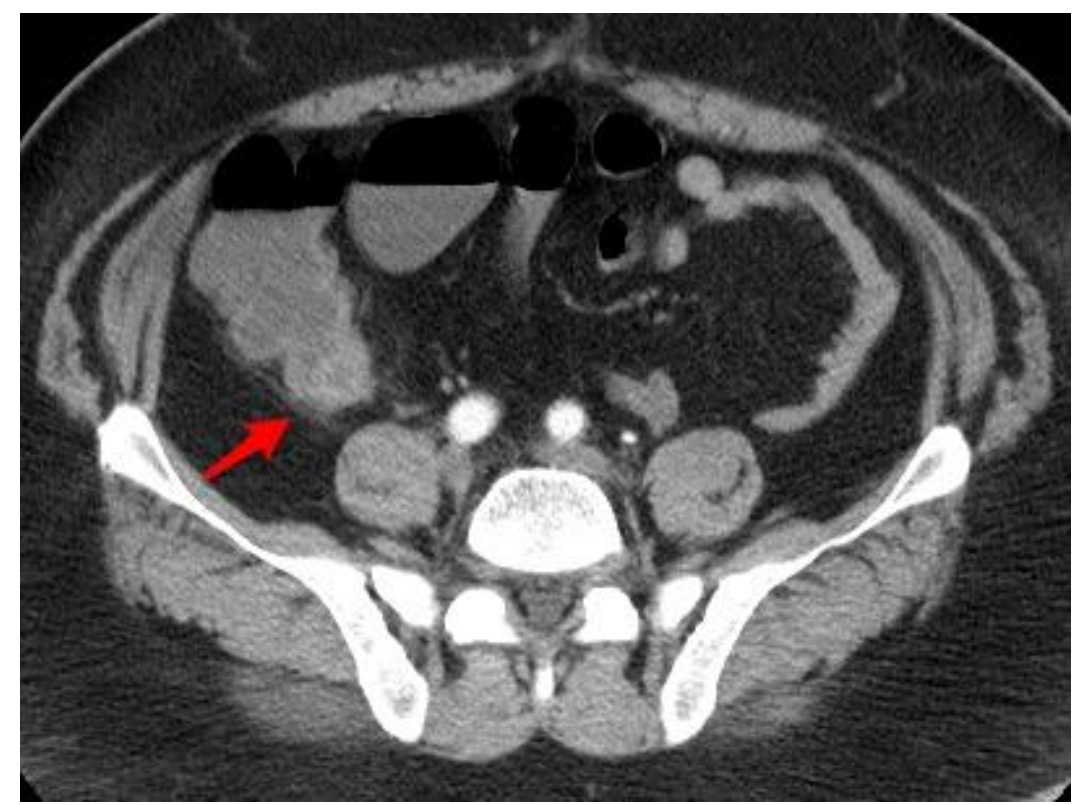

Fig (4):- CT abdomen and Pelvis, axial view, arterial phase: Focal mural thickening at the tip of the cecum/root of the appendix with mucosal hyperenhancement (red arrow).

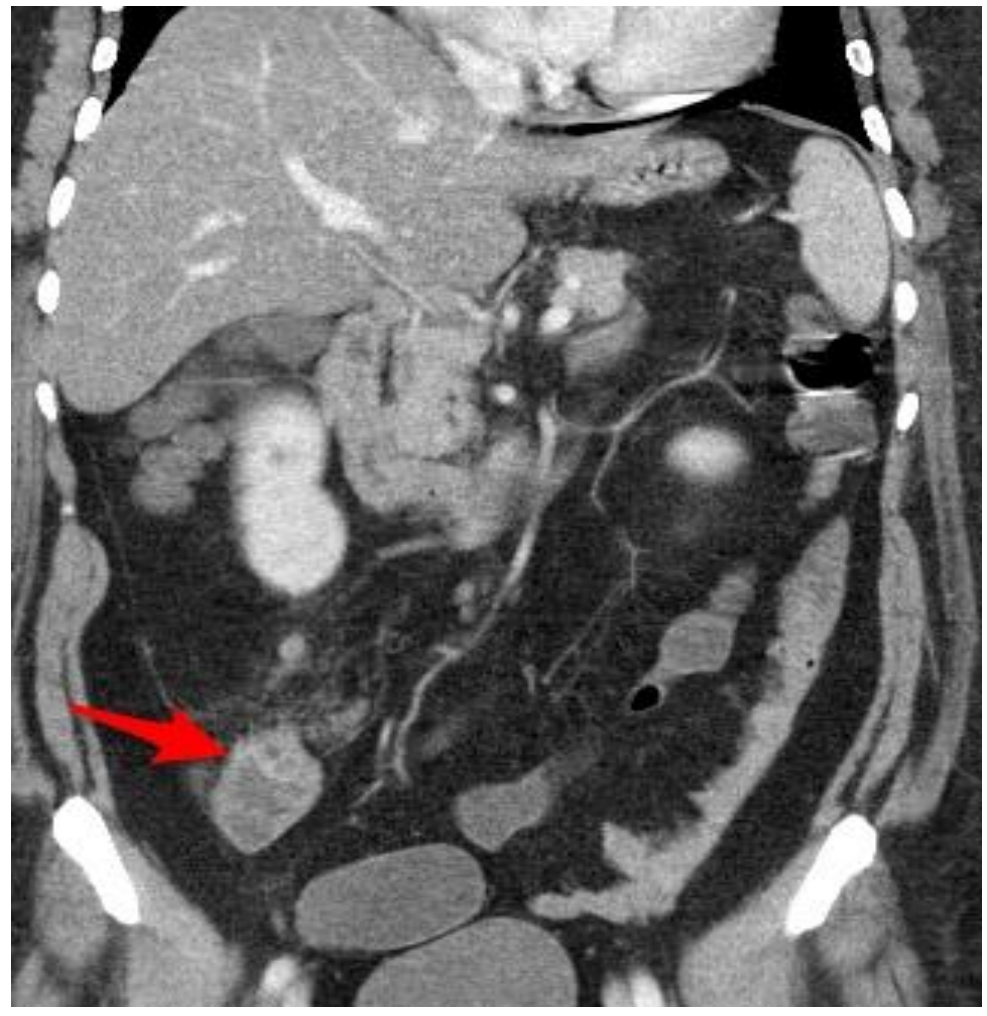

Fig (5):- CT abdomen and Pelvis, Coronal Reformat, portal venous phase: Focal mural thickening at the tip of the cecum/root of the appendix with mucosal hyper enhancement (red arrow) 


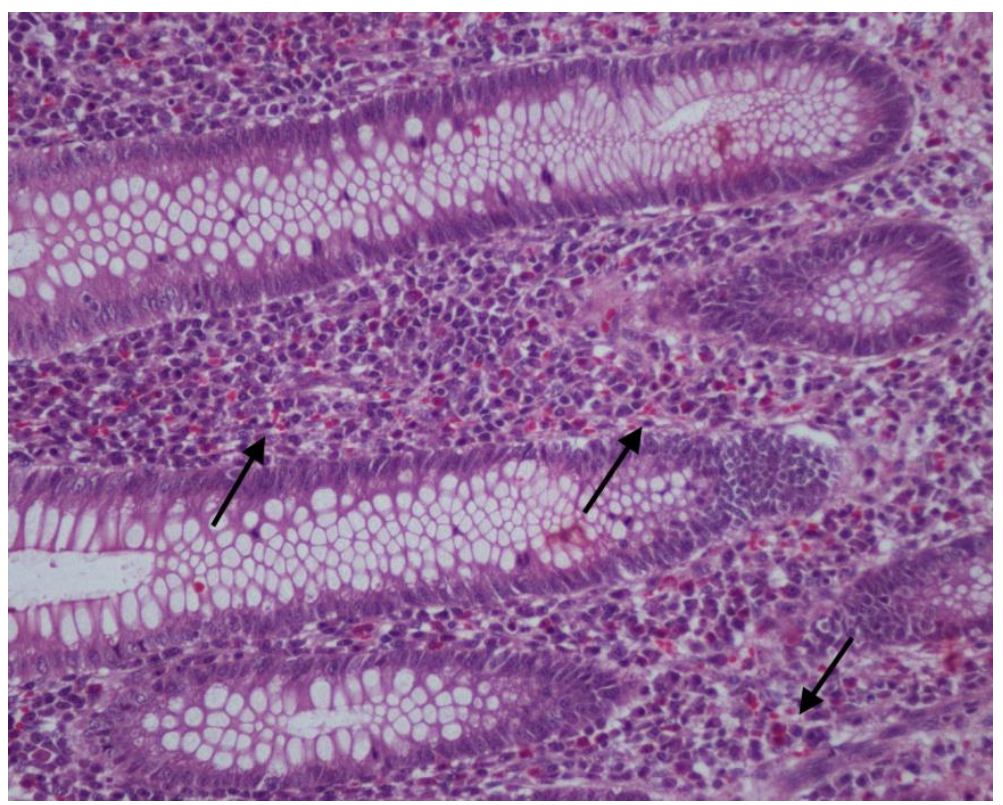

Fig (6):- Dense mucosal infiltration by eosinophilic (black arrows) H\&E X20.

\section{Discussion:-}

Acute eosinophilic appendicitis was proposed in 1996 for the first time by Aravindan; then it was defined in 2010 by Aravindan et al. They reported that an allergic reaction type 1 hypersensitivity is a predisposing event, which triggers the acute inflammation of the appendix. This inflammation involves muscularispropria eosinophilic infiltration and edema [5], both are considered the hallmarks of this disease entity [8].

Type 1 hypersensitivity is not only developed in the appendix but also in other adjacent areas such as ileum and cecum, whereas the appendix considered the most affected organ by allergic reaction [4]. Although eosinophils are normally found in submucosal layer and lamina propria of the appendix, evidence of infiltration through the muscularispropria combined with edema should raise the suspicion of acute eosinophilic appendicitis [2].

The presentation of acute eosinophilic appendicitis is not different from the classic acute appendicitis, except in AEA, the symptoms are less in duration [2]. In our case report, the patient has presented mainly by acute LGIB, which is a rare presentation of acute appendicitis [6]. Magaz MM, et al, reported 30 cases that presented by bleeding from the appendix none were due to eosinophilic infiltration [10]. To our knowledge, this is the first case of acute eosinophilic appendicitis presenting with LGIB.

Many case reports have used different modalities such as colonoscopy, radionuclide scintigraphy, angiography, and multi-detector computed tomography (MDCT) to diagnose appendicular bleeding [11]. The combination of endoscopy with radiology may increase the accuracy of diagnosis during acute bleeding phase [6].

Management of appendiceal hemorrhage depends on patient's general condition and underlying etiology, and surgical intervention play a major role in treating appendiceal lesions and bleeding including laparoscopic versus open appendectomies, extensive resection of the bowel, and right hemicolectomy. In this case right hemicolectomy was done, as in most cases removal of the cecum in needed to control the bleeding [6].

In order, to reach definitive diagnosis histopathological features are needed which include: abundant eosinophilic infiltration in muscularispropria rather than neutrophil infiltration. This should be associated with edema [4].

According to this case, histopathology examination of the specimen revealed acutely inflamed appendix, mucosal ulceration, transmuraleosinophils infiltrate with no granulomatous formation or malignant changes. 


\section{Conclusion:-}

Acute Eosinophilic Appendicitis (AEA) is a rare and not well-studied disease and it can be present with unusual symptom as LGIB. It is important to conduct further studies about AEA, for better diagnosis approach and management, as well as understanding the pathogenesis of this entity.

\section{References:-}

1. Apandisit AE, Enflamasyonunun A, Tipi NB. Acute Eosinophilic Appendicitis: An Unusual Variant of Appendix Inflammation.

2. Shrestha R, Shrestha A, Tiwari M, Ranabhat S, Maharjan S. Role of Eosinophils in Acute Appendicitis. Journal of Nepal Medical Association. 2015;53(197):12-7.

3. Aravindan KP, Vijayaraghavan D, Manipadam MT. Acute eosinophilic appendicitis and the significance of eosinophil-Edema lesion.Indian Journal of Pathology and Microbiology. 2010 Apr 1;53(2):258.

4. Singh UR, Malhotra A, Bhatia A. Eosinophils, mast cells, nerves and ganglion cells in appendicitis. Indian Journal of Surgery. 2008 Oct 1;70(5):231-4.

5. Kumbar RK, Dravid NV, Karibasappa GN, Surana A. Acute Eosinophilic Appendicitis: Case report of three cases with brief review of literature. Annals of Applied Bio-Sciences. 2015 Jun 5;2(2):C18-21.

6. Chiang CC, Tu CW, Liao CS, Shieh MC, Sung TC. Appendiceal hemorrhage-An uncommon cause of lower gastrointestinal bleeding. Journal of the Chinese Medical Association. 2011 Jun 30;74(6):277-9.

7. Kolur A, Patil AM, Agarwal V, Yendigiri S, Sajjanar BB. The Significance of Mast Cells and Eosinophils Counts in Surgically Resected Appendix. Journal of Interdisciplinary Histopathology. 2014;2(3):150-3.

8. Magaz MM, DE LA Revilla NJ, Martín LJ, Gonzalez PI, DE Las Heras T, Sanchez YM, Ríos GR, Salas AC, Abreu GL. Appendicular bleeding: An exceptionalcause of lower hemorrhage. Revistaespanola de enfermedadesdigestivas: organooficial de la Sociedad Espanola de PatologiaDigestiva. 2015 Jul;107.

9. Chung KS, GaoJP.Massive lower gastrointestinal bleeding from the appendix. Gut and liver. 2011 Jun;5(2):234. 